\title{
Nonlocal modelling of superelastic behavior of shape memory alloys
}

\author{
Arnaud Duval ${ }^{\mathrm{a}}$, Mohamed Haboussi, and Tarak Ben Zineb \\ LEMTA, Nancy-University, CNRS, 2 rue Jean Lamour, 54519 VANDOEUVRE lès Nancy CEDEX, \\ France
}

\begin{abstract}
Recently, various constitutive laws based on phenomenological or micromechanical approaches have been developed to describe the behavior of shape memory alloys (SMA). They are particularly well adapted for modelling the effects of phase transformation and martensite reorientation on the thermomechanical behavior of the bulk material.

Nevertheless, these models fail to describe phenomena such as transformation localisation or size effects which may appear in wires and thin films. Indeed, to describe such phenomena, the influence of the neighbouring area of the material point should be taken into account. This can be achieved through a nonlocal formulation of the constitutive equations.

In the present work, we propose a nonlocal modelling of the superelastic behavior of SMA. It uses for the martensite volume fraction, besides the usual local variable, a corresponding nonlocal contribution. This latter is governed by a partial differential equation defined on an influence zone whose size is dependant on an internal length parameter of the material.

Based on this formulation, a 1D specific element is developped where the nonlocal variable is an additional degree of freedom. Simulations are performed to study the influence of the internal length parameter and nonlocality on the mechanical response during superelastic loadings.
\end{abstract}

\section{Introduction}

Shape Memory Alloys are exhibiting behavior that made them well adapted for the design of small-sized devices as micro-actuators. The ratio between mechanical work and response time made them more suitable for microfluidic applications such as micropumps compared to piezoelectric ceramics [1] [2]. If the behavior of bulk SMA is well described by macroscopic models adopting a local approach [3] [4] [5], a size effect is present for small samples as wires or thin films that can not be taken into account by the cited models. At this scale, a strain localisation due to the instability of martensitic phase transformation is observed [6] [7]. This localisation strongly depends on the neighbouring area of the considered point.

A model able to describe this behavior is proposed, based on a nonlocal description of the martensite volume fraction. A non-local variable is defined and approximated by a gradient formulation. A simple constitutive model describing the local behavior of the material and taking into account the neigbouring area defined by an internal length scale linked to the microstructure is written. It is based on Peultier's approach [5] and describes the superelastic behavior. The gradient formulation is implemented in the finite element code ABAQUS through the development of a $1 \mathrm{D}$ specific element where the nonlocal variable is considered as an additional degree of freedom.

\footnotetext{
a e-mail: arnaud.duval@esstin.uhp-nancy.fr
} 
Several numerical simulations are performed to study the influence of the internal length on the material response.

\section{Definition of a nonlocal variable}

The aim of the proposed approach is to describe the superelastic behavior of SMAs. It can be easily described by a unique internal variable, the local martensite volume fraction $f$. To take into account the neighbouring of the considered material point, a nonlocal volume fraction is usually defined as [8] :

$$
\bar{f}(x)=\int_{\Omega} G(y, x) f(y) \mathrm{d} \Omega(y)
$$

This expression exhibits a Green-type function $G$ which can take the following expression proposed by Zauderer [9] :

$$
G(x, y)=\frac{1}{4 \pi \rho \ell^{2}} \exp \left(\frac{\rho}{\ell}\right)
$$

where $\rho=|x-y|$ The integral (Eq. 1) can be approximated by introducing a Taylor developpement of the local variable $f$ around a position $x$ :

$$
\begin{aligned}
f(y)= & f(x)+\frac{\partial f}{\partial x}(y-x)+\frac{1}{2 !} \frac{\partial^{2} f}{\partial x^{2}}(y-x)^{2}+\frac{1}{3 !} \frac{\partial^{3}}{\partial x^{3}}(y-x)^{3} \\
& +\frac{1}{4 !} \frac{\partial^{4} f}{\partial x^{4}}(y-x)^{4}+\ldots
\end{aligned}
$$

Substituting (3) in (1), this latter can be rewritten under the form :

$$
\bar{f}(x)=f(x)+c(\ell) f_{, x x}(x)+d(\ell) f_{, x x x x}(x)+\ldots
$$

after taking into account of the symmetry properties of $G$.

Equation 4 is an explicit definition because the nonlocal quantity $\bar{f}$ can be directly deduced from the local quantity $f$. But according to Peerlings et al. [10], this type of formulation corresponds to a weak nonlocality. It can be improved by taking into account higher orders of the Taylor approximation which may lead to a very high numerical cost. Another way of improvment is obtained by considering the second derivative of equation 4 :

$$
\bar{f}_{, x x}(x)=f_{, x x}(x)+c(\ell) f_{, x x x x}(x)+\ldots
$$

The obtained quantity is multiplied by $c(\ell)$ and substracted to equation 4 :

$$
\bar{f}(x)-c(\ell) \bar{f}_{, x x}(x)=f(x)+\left(d(\ell)-c^{2}(\ell)\right) f_{, x x x x}+\ldots
$$

Engelen et al. [11] showed that the adopted $G$ function (Eq. 2) implies $d(\ell)-c^{2}(\ell)=0$ and $c(\ell)=\ell^{2}$ for an arbitrary field $f(x)$. The higher order terms of Eq. 6 can be neglected if the proposed Green function is adopted as a ponderation function. It can be noted that the local field is recovered when $\ell \rightarrow 0(\bar{f}=f)$. The implicit definition of the nonlocal variable $\bar{f}$ reads :

$$
\bar{f}(x)-c(\ell) \bar{f}_{, x x}(x)=f(x)
$$

A Neumann boundary condition is specified at the domain border:

$$
\bar{f}_{x}=0 \quad \text { on } \quad \Gamma
$$

Which induces :

$$
\int_{\Omega} \bar{f} \mathrm{~d} \Omega=\int_{\Omega} f \mathrm{~d} \Omega
$$

This condition ensures that the total quantity of martensite is conserved inside the domain $\Omega$. 


\section{Application to a superelastic behavior}

The aim is to validate the nonlocal approach in the framework of SMA modelling. A simple $1 \mathrm{D}$ superelastic model is considered. It has only one internal variable : the martensite volume fraction $f$. According to Peultier's model [5], a single driving force associated to the phase transformation process can be defined :

$$
F_{f}=\Sigma-B\left(T-T_{0}\right)-H_{f} f-\alpha_{0} \frac{f-1}{f}-\alpha_{1} \frac{f}{1-f}
$$

Where $\Sigma$ is the uniaxial stress, $B$ the stress-temperature ratio, $T$ the testing temperature, $T_{0}$ the balance temperature of the martensitic transformation, $H_{f}$ the pseudo-hadrening coefficient corresponding to martensitic transformation. The two last terms ensure the condition $0 \leq f \leq 1$ by penalty.

The dissipative process which corresponds to the phase transformation is represented by a dry friction, introducing a critical value $F_{f}^{c r i t}$ :

$$
\left|F_{f}\right| \leq F_{f}^{c r i t}
$$

Where $F_{f}^{c r i t}$ can decrease from its initial value $F_{f 0}^{c r i t}$ during the localisation of martensitic transformation :

$$
F_{f}^{c r i t}=F_{f 0}^{c r i t} e^{-H_{\bar{f}} \bar{f}}
$$

The local total strain $E$ can be expressed as :

$$
E=\frac{\Sigma}{Y}+E^{T}
$$

where $Y$ is the young modulus assumed to be the same in both phases and $E^{T}$, the transformation strain defined by :

$$
\dot{E}^{T}=\dot{f} N \varepsilon_{\text {sat }}^{T}
$$

where $\varepsilon_{\text {sat }}^{T}$ is the saturation value of the transformation strain and $N$ the orientation direction of the created martensite. It is assumed to be equal to the stress direction.

The presented behavior law has two control variables : the total strain $E$ and the nonlocal martensite volume fraction $\bar{f}$. The balanced equations can be formulated under the incremental form by introducing the tangent operators $H^{u u}, H^{u \bar{f}}, \ldots$ :

$$
\left\{\begin{array}{l}
\delta \Sigma=H^{u u} \delta E+H^{u \bar{f}} \delta \bar{f} \\
\delta f=H^{\bar{f} u} \delta E+H^{\overline{f f}} \delta \bar{f}
\end{array}\right.
$$

\section{Weak formulation of equilibrium}

Equilibrium at a material point can be defined by a set of two constitutive equations :

$$
\left\{\begin{array}{l}
\Sigma_{, x}=0 \\
f=\bar{f}-\ell^{2} \bar{f}_{, x x} \quad \text { on } \quad \Omega
\end{array}\right.
$$

The first one is the mechanical equilibrium condition in the static situation where body forces are assumed to be zero. The second equation derived from Eq. 7 takes into account the definition of the nonlocal variable. Two virtual fields are introduced :

$$
\begin{aligned}
& W_{u}=\left\{w_{u} \backslash w_{u} \in\left[C^{0}\right]\right\} \\
& W_{\bar{f}}=\left\{w_{\bar{f}} \backslash w_{\bar{f}} \in\left[C^{0}\right]\right\}
\end{aligned}
$$


$w_{u}$ and $w_{\bar{f}}$ are associated to the displacement the nonlocal martensite volume fraction respectively. Equilibrium equation is mutiplied by the virtual field and integrated on the whole domain $\Omega$ :

$$
\int_{\Omega} w_{u} \Sigma_{, x} \mathrm{~d} \Omega=0
$$

The partial integration of (18) gives :

$$
\int_{\Omega} w_{u, x} \Sigma \mathrm{d} \Omega=\int_{\Gamma} w_{u} T \mathrm{~d} \Gamma \quad \forall w_{u} \in W_{u}
$$

where $T$ corresponds to the stress conditions appliedto the domain boundaries. For the nonlocal martensite volume fraction, the following relation is obtained after introducing the Neumann boundary condition :

$$
\int_{\Omega} w_{\bar{f}} \bar{f}+\ell^{2} w_{\bar{f}, x} \bar{f}_{, x} \mathrm{~d} \Omega=\int_{\Omega} w_{\bar{f}} f \mathrm{~d} \Omega
$$

Variables $\Sigma$ and $f$ are linearized around the increment $(i)$ :

$$
\begin{aligned}
\Sigma & =\Sigma^{(i-1)}+\delta \Sigma \\
f & =f^{(i-1)}+\delta f
\end{aligned}
$$

Introduction of the local tangent operators defined in Eq. 15 leads to:

$$
\int_{\Omega} w_{u, x} H^{u u} \delta E \mathrm{~d} \Omega+\int_{\Omega} w_{u, x} H^{u \bar{f}} \delta \bar{f} \mathrm{~d} \Omega=-\int_{\Omega} w_{u, x} \Sigma^{(i-1)} \mathrm{d} \Omega+\int_{\Gamma} w_{u} T^{(i)} \mathrm{d} \Gamma
$$

and

$$
\begin{aligned}
& -\int_{\Omega} w_{\bar{f}}\left(H^{\bar{f} u} \delta E+H^{\overline{f f}} \delta \bar{f}\right) \mathrm{d} \Omega+\int_{\Omega}\left(w_{\bar{f}} \delta \bar{f}+\ell^{2} w_{\bar{f}, x} \delta \bar{f}_{, x}\right) \mathrm{d} \Omega \\
= & \int_{\Omega} w_{\bar{f}} f^{(i-1)} \mathrm{d} \Omega-\int_{\Omega}\left(w_{\bar{f}} \bar{f}^{(i-1)}+\ell^{2} w_{\bar{f}, x} \bar{f}_{, x}^{(i-1)}\right) \mathrm{d} \Omega
\end{aligned}
$$

\section{Spatial discretisation}

In order to solve the partial derivative equations resulting from the weak formulation of equilibrium, a spatial discretisation in finite elements must be introduced. Thus, the displacement and nonlocal martensite volume fraction fields are discretized at each node of the finite element mesh, in respectively vector $\left\{u^{N}\right\}$ and $\left\{\bar{f}^{N}\right\}$. A reference element is introduced, whose coordinate $\xi$ belongs to $[-1: 1]$. Interpolation matrices $\left[N_{N}^{u}\right]$ and $\left[N_{N}^{\bar{f}}\right]$ lead to the expressions of $u$ and $\bar{f}$ at a given coordinate $\xi$ :

$$
\begin{array}{rll}
u(\xi)=\left[N_{N}^{u}\right]\left\{u^{N}\right\} & \text { with } & {\left[N_{N}^{u}\right]=\left[\frac{1-\xi}{2} \frac{1+\xi}{2}\right]} \\
\bar{f}(\xi)=\left[N_{N}^{\bar{f}}\right]\left\{\bar{f}^{N}\right\} & \text { with } & {\left[N_{N}^{\bar{f}}\right]=\left[\frac{1-\xi}{2} \frac{1+\xi}{2}\right]}
\end{array}
$$

Operators $\left[B_{N}^{u}\right]$ and $\left[B_{N}^{\bar{f}}\right]$ are adopted to compute the value of strain and gradient of nonlocal martensite volume fraction :

$$
\begin{array}{rrr}
E(\xi)=\left[B_{N}^{u}\right]\left\{u^{N}\right\} & \text { with } & {\left[B_{N}^{u}\right]=[-1 / L 1 / L]} \\
\bar{f}_{, x}(\xi)=\left[B_{N}^{\bar{f}}\right]\left\{\bar{f}^{N}\right\} & \text { with } & {\left[B_{N}^{\bar{f}}\right]=[-1 / L 1 / L]}
\end{array}
$$




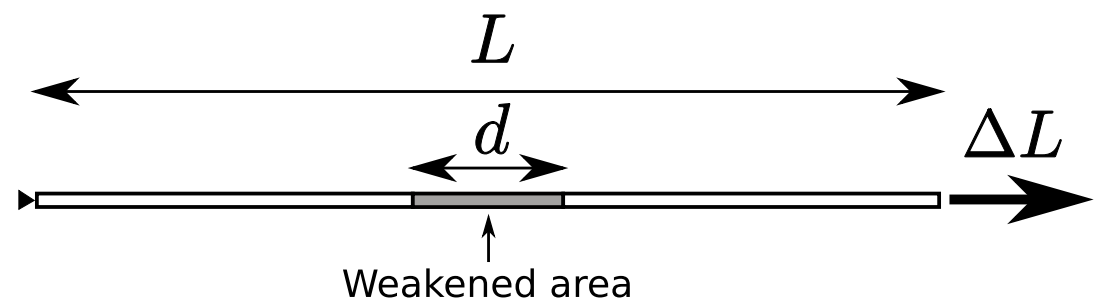

Fig. 1. Tension test on a weakened truss.

In the relation (25), $L$ is the meshing size. Interpolation matrices and gradient operators are introduced into the weak formulation of equilibrium (Eqs. 22 and 23). As a result, the infinitesimal increments of displacement and nonlocal martensite volume fraction can be expressed as a function of stiffness sub-matrices and nodal residual forces :

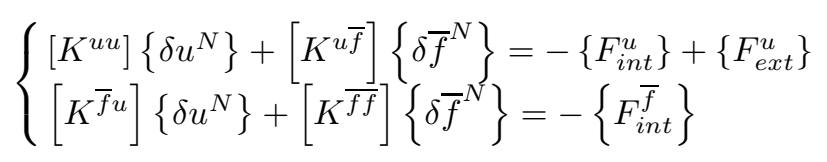

Stiffness matrices and residual forces are expressed as integrals on the element domain $\Omega_{e}$. As an example, the submatrix $K^{\overline{f f}}$ is written :

$$
\begin{aligned}
{\left[K^{\overline{f f}}\right] } & =\int_{\Omega_{e}}\left[N_{N}^{\bar{f}}(\xi)\right]^{T}\left(1-H^{\overline{f f}}(\xi)\right)\left[N_{N}^{\bar{f}}(\xi)\right] \mathrm{d} \Omega+\int_{\Omega_{e}} \ell^{2}\left[B_{N}^{\bar{f}}(\xi)\right]^{T}\left[B_{N}^{\bar{f}}(\xi)\right] \mathrm{d} \Omega \\
& =\int_{-1}^{1}\left[N_{N}^{\bar{f}}(\xi)\right]^{T}\left(1-H^{\overline{f f}}(\xi)\right)\left[N_{N}^{\bar{f}}(\xi)\right] \mathcal{J} \mathrm{d} \xi+\int_{-1}^{1} \ell^{2}\left[B_{N}^{\bar{f}}(\xi)\right]^{T}\left[B_{N}^{\bar{f}}(\xi)\right] \mathcal{J} \mathrm{d} \xi
\end{aligned}
$$

The numerical integration is achieved, based on the Gauss quadrature method where two points of integration are considered. The equation 27 becomes :

$$
\left[K^{\overline{f f}}\right]=\sum_{i=1}^{2} w_{i}\left[N_{N}^{\bar{f}}\left(\xi_{i}\right)\right]^{T}\left(1-H^{\overline{f f}}\left(\xi_{i}\right)\right)\left[N_{N}^{\bar{f}}\left(\xi_{i}\right)\right] \mathcal{J}+\sum_{i=1}^{2} w_{i} \ell^{2}\left[B_{N}^{\bar{f}}\left(\xi_{i}\right)\right]^{T}\left[B_{N}^{\bar{f}}\left(\xi_{i}\right)\right] \mathcal{J}
$$

where $\mathcal{J}$ is the determinant of the jacobian matrix corresponding to the tranformation from reference element to spatial element $(\mathcal{J}=L / 2) . \xi_{i}$ and weight $\omega_{i}$ are the weight and coordinate of the integration point.

\section{Implementation and simulations}

The developed element is implemented into the finite element code ABAQUS via the user subroutine UEL. This routine allows the user to define its own finite element by programming stiffness matrices and residual nodal forces corresponding to an increment of the degrees of freedom must be computed.

Simulations are made on a truss whose central zone is weakened by taking into account a reduced section area (see Fig. 1). The parameters concerning the material and the structure used in these simulations are given in Tab. 1 . Several simulations are made to study the influence of the internal length parameter $\ell$ and softening parameter $H_{\bar{f}}$ on the material response.

In the Fig. 2 we report the reaction force response of the weakened truss as a function of the applied displacement. It can be decomposed as the following different steps :

- Elastic behavior

- Start of martensitic transformation 

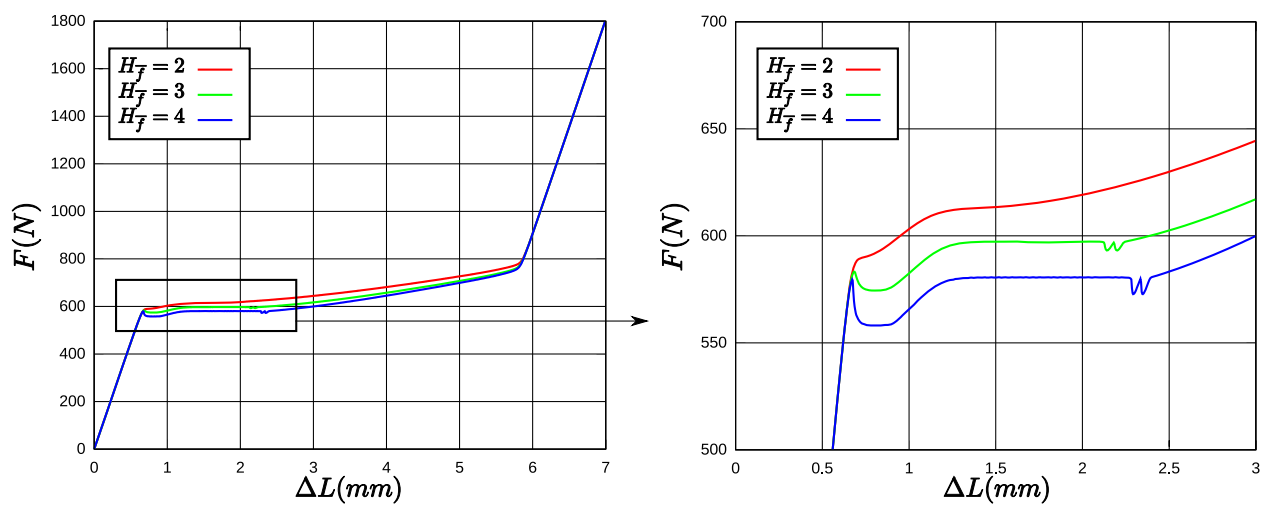

Fig. 2. Force-displacement response of a weakened truss for several value of parameter $H_{\bar{f}}(\ell=1)$.

\begin{tabular}{cc}
\hline$L$ & $100 \mathrm{~mm}$ \\
$d$ & $20 \mathrm{~mm}$ \\
$S$ & $1.3 \mathrm{~mm}^{2}$ \\
$S_{\text {weakened }}$ & $1.25 \mathrm{~mm}^{2}$ \\
$Y$ & $70000 \mathrm{MPa}$ \\
$B$ & $5 \mathrm{MPa} /{ }^{\circ} \mathrm{C}$ \\
$T$ & $25^{\circ} \mathrm{C}$ \\
$T_{0}$ & $-40^{\circ} \mathrm{C}$ \\
$F_{f 0}^{c r i t}$ & $150 \mathrm{MPa}$ \\
$H_{f}$ & $250 \mathrm{MPa}$ \\
$\varepsilon_{\text {sat }}^{T}$ & 0.05 \\
$\alpha_{0}$ & 0.1 \\
$\alpha_{1}$ & 0.1 \\
\hline
\end{tabular}

Table 1. Structural and material parameters

- Softening due to the localization of the phase transformation in the weakened area

- Phase transformation into the weakened zone

- Propagation of phase transformation through the whole structure

- Fully martensitic structure with elastic behavior

Simulations show that the internal length parameter has an influence on the space propagation of the martensitic phase transformation (see Fig. 3). This phenomena may be linked to the one observed by Sun [7]. In a material exhibiting small grains, grain boundary volume fraction can not be neglected and tends to slown down the propagation of martensitic transformation. On the opposite side, the martensite variants can easilly grow up in big grain. Further investigations have to be made in order to establish a constitutive relation between grain size and internal length scale $\ell$.

\section{Conclusion}

A 1D finite element was developped to take into account the localisation effects in shape memory alloy wires. The instability of the behavior law adopted is regularized by introducing a non local variable $\bar{f}$ which is treated as an additional degree of freedom. Further development will focus on the implementation of a $2 \mathrm{D}$ finite element adapted for the design of a microfluidic pump made of SMA thin films. As for the volume fraction, the taking account of the nonlocality of the martensite orientation variable may improve our modelling of shape memory effect. 

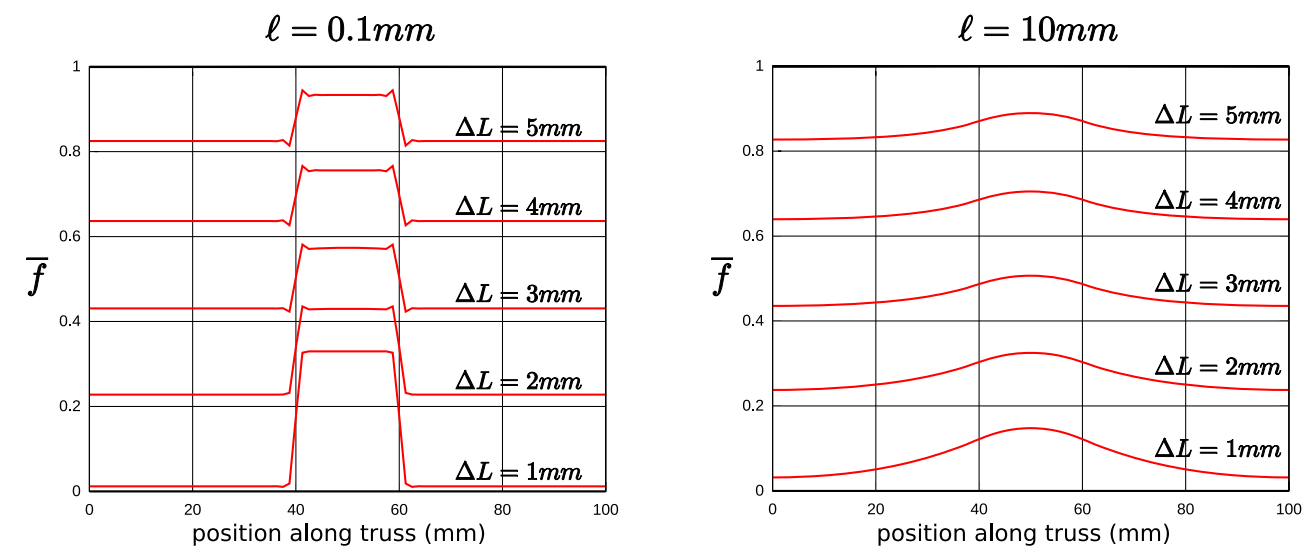

Fig. 3. Influence of parameter $\ell$ on the propagation of phase transformation.

\section{References}

1. W.L. Benard, H. Kahn, A.H. Heuer, M.A. Huff, Thin film shape memory alloy actuated micropumps, J. MEMS 7, (1998) 245-251.

2. M. Koch, N. Harris, A.G.R. Evans, N.M. White, A. Brunnschweiler, A novel micromachined pump based on thick film piezoelectric actuation, Sensors and Actuatrs A, 70, (1998) 98-103.

3. D. Lagoudas, P. Entchev, Modeling of the transformation induced plasticity and its effects on the behavior of porous shape memory alloys. Part I : constitutive model for fully dense SMAs, Mechanics of Materials 38, (2004) 865-892.

4. F. Thiebaud, C. Lexcellent, M. Collet, E. Foltete, Implementation of a model taking into account the asymmetry between tension and compression, the temperature effects in a finite element code for shape memory alloys structures calculations, Computational Materials Sciences 41, (2007) 208-221.

5. B. Peultier, T. Ben Zineb, E. Patoor, Macroscopic constitutive law for shape memory alloy thermomechanical behaviour. Application to structure computation by FEM, Mechanics of Materials 38, (2006) 510-524.

6. C.B. Churchill, J.A. Shaw, M.A. Iadicola, Tips and tricks for characterizing shape memory alloy wire: Part 2-Fundamental isothermal responses, Experimental Techniques 33, (2009) 51-62.

7. Q.P. Sun, Y.J. He, A multiscale continuum model of the grain-size dependance of the stress hysteresis in shape memory alloy polycrystals, International Journal of solids and Structure 45, (2008) 38683896.

8. G. Pijaudier-Cabot, Z. P. Bažant, Nonlocal damage theory, Journal of Engineering Mechanics-ASCE 113, (1987) 1512-1533.

9. E. Zauderer, Partial differential equations of applied mathematics, Wiley, Chichester, UK, 2nd edition (1989).

10. R.H.J. Peerlings, R. De Borst, W.A.M. Brekelmans, Wave propagation and localisation in non-local gradient enhanced damage models, Journal de Physique IV 8, (1998) 293-300.

11. R.A.B. Engelen, M.G.D. Geers, F.P.T. Baaijens, Nonlocal implicit gradient-enhanced elastoplasticity for the modelling of softening behaviour, International journal of Plasticity 19, (2003), 403-433. 